\title{
Rabies wonderful Strategies to Escape the Immune System
}

\author{
Mahsa Taherizadeh ${ }^{1}$, Rouzbeh Bashar ${ }^{1}$, Mahsa Golahdouz ${ }^{1}$, Behzad Pourhossein ${ }^{2}$ and Maryam Fazeli1* $^{1 *}$ \\ ${ }^{1}$ Virology Department, Pasteur institute of Iran, Tehran, Iran \\ ${ }^{2}$ Department of Virology, School of Public Health, Tehran University of Medical Sciences, Tehran, Iran
}

Submission: December 22, 2018; Published: March 25, 2019

*Corresponding author: Maryam Fazeli, National Center for Reference and Research on Rabies, Virology Department, Pasteur Institute of Iran, 12 Farvardin St, Tehran, Iran

\begin{abstract}
Rabies infection is one of the fatal zoonotic encephalitis and caused by neurotropic viruses in the genus Lyssavirus, family Rhabdoviridae. Rabies virus is unique because the virus replicates in nervous system and sheds from saliva when its host is stimulated to bite. The virus escapes from responses of the immune system with some mechanisms as much as possible. So far, many studies have been conducted to identify the methods of escaping the virus from the immune system. This mini-review study discusses about some distraction rabies strategies to escape from immune system.

Abbrevations: BBB: Blood-Brain Barrier; CMI: Cell-Mediated Immune; CHUK: Conserved Helix-Loop-Helix Ubiquitous Kinase; CNS: Central Nervous System; G: the Rabies virus Glycoprotein; IFN-1: Interferon Type I; IKBKB: Inhibitor of Kappa Light Polypeptide Gene Enhancer in B-cells, Kinase beta; IRF-9: Interferon Regulatory Factor-9; ISG: Interferon-Stimulated Gene; ITK: IL2-Inducible T-cell kinase; JAK: Janus Kinase; MAVs: Mitochondria Antiviral Signaling Protein; MYD88: Myeloid Differentiation Primary Response 88; NCAM: Neuronal Cell Adhesion Molecule;

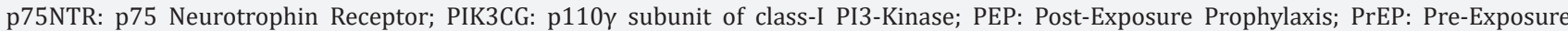
Prophylaxis; PRRs: Pathogen Recognition Receptors; RAF: Rapidly Accelerated Fibrosarcoma; RV: Rabies Virus; RLR: The RIG-like Receptor; ssRNA: Single-Stranded RNA; STAT: Signal Transducers and Activator of Transcription; TBK-1: Serin-Threonine Protein Kinase-1; TLRs: Toll-Like Receptors
\end{abstract}

\section{Introduction}

Rabies virus (RV) causes a zoonotic and fatal infectious disease among all warm-blooded animals including humans that already do not acquire immunity against rabies [1,2]. Rabies virus as a negative-sense, single-stranded RNA (ssRNA) virus is a type species of the Lyssavirus genus of the Rhabdoviridae family. Rabies is cause of more than 59,000 deaths annually worldwide. The disease is endemic in most parts of the world, especially in Asia and Africa. In addition, this virus is capable of infecting livestock and creating financial damage [3-5]. Therefore, it is one of the major health problems in every community. So far, many studies have been performed to identify the methods of escaping the virus from the immune system. This mini-review study discusses about some distraction rabies strategies to escape from immune system.

\section{Pathogenesis of Rabies}

Infected animals carrying the virus in their saliva and in the final stages of the disease they transmit the virus to other sensitive animals or humans usually via biting. Unusual transfer methods such as organ transplant and aerosol (for example in infested bat caves) have also been reported in some human rabies cases [6]. At the first, the viruses replicate in myocytes, at the wound site [7] Therefore, viruses reach to the brain via reverse axonal transport and finally encephalitis occurs. Rabies viruses have a different pathogenesis than other viruses, the virus enters to central nervous system (CNS) by escaping the immune system and causes pathogenesis [8]. The infection incubation period is varying from around 7 days to 3 months. Significant variance in the incubation period occurs due to difference in amount of the virus which introduced to the wound, severity and distance of the wound to CNS [9]. Direct inoculation of the virus into the nerve leads to a shortening of incubation period due to presence of several bite at different areas such as the neck and head [9].

Several molecules have been described on the nerve cells surface, which need to facilitate the virus entry into the cells as receptors such as the muscular family of nicotinic acetylcholine receptor (nAChR), neuronal cell adhesion molecule (NCAM), and p75 neurotrophin receptor (p75NTR) (Figure 1). The nAChR is the most important receptor for RV to enter to neuromuscular system [10]. Differences in these receptors affect the sensitivity of different 
species to the rabies. The axonal transport occurs at a rate of 12 to $24 \mathrm{~mm}$ per day. On the other hand, the rapid axonal movement by microtubule-dependent motors and slow axonal movement via actions are mediated in this transport. Laboratory experiments showed the Colchicine effect on the virus axonal movement by destruction of microtubule factors. As a result, the drug leads to a slow transportation during axon that can effectively prevent spread of the virus [11]. As the virus reaches to CNS, neurological signs appear in two forms: classic encephalitic (furious) rabies or paralytic (dumb) rabies. Furious rabies, which occurs in $80 \%$ of patients, is well recognized with aphasia, incoordination, paresis, paralysis, hydrophobia, aerophobia, larynx spasms, confusion, delirium, hallucinations, hyperactivity to aerophobia and seizures [12]. Fourteen days after the onset of symptoms, death occurs due to cardiac arrhythmia and cardiopulmonary arrest [13].
In dumb rabies, an early sign is organ weakness with no loss of consciousness. This weakness begins at the site of the bite and spreads rapidly to other organs. Finally, paralysis spreads to the respiratory system and can cause death by asphyxia or choking. Patients can survive longer with dumb rabies than with furious rabies, persevering for up to 30 days [14].

The pathogenicity of rabies virus depends on the presence of an antigenic determinant on the viral glycoprotein (G) (for example, arginine or lysine position in 333aa of two different strains (ERA and CVS)). The glycoprotein is essential for integrity of an antigenic determinant and rabies virulence in adult mice. In addition, the lysine mutation at position 330 of the glycoprotein destroys virus infiltration into motor-sensory neurons after muscle inoculation [15].
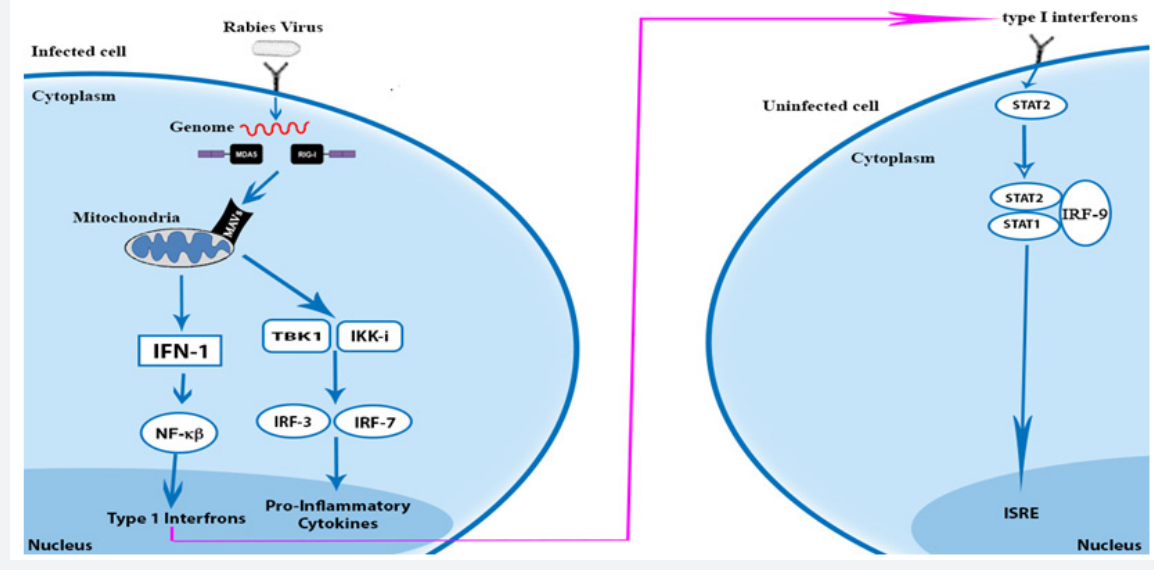

RIG-1: Retinoic Acid-Inducible Gene-1, MAVs: Mitochondria Antiviral Signaling Protein, TBK-1: Serin-Threonine Protein Kinase-1, IRF: Interferon Regulatory Factor, IKK: IKB kinase, IFN: interferon, JAK-1: Janus Kinase-1, STAT: Signal Transducers and Activator of Transcription.

Figure 1: Schematic diagram of the IFN signaling pathways induced by the RV.

\section{Intrinsic Immunity Performance}

First defense line in body against the pathogenic factor is the intrinsic immunity. The intrinsic immunity cells produce different cytokines such as interferon $\alpha / \beta$ and chemokines, which leads to activation of the complement and absorption of phagocytes including neutrophils and macrophages to the site of infection [16]. According to numerous studies, toll-like receptors (TLRs) are the best pathogen recognition receptors (PRRs) as part of the intrinsic immune response. Toll-like receptors expression in neurons has not been reported in normal conditions, until now. However, it has been observed that the human neurons express the TLR-3 molecule when are infected with some viruses such as RV. Beta interferon, chemokines and inflammatory cytokines are produced from infected cells by stimulating this receptor. The studies indicate that in rabies-infected neurons, the receptor contributes to development of some inclusion bodies (IBs). These IBs consist of viral $\mathrm{N}$ and $\mathrm{P}$ proteins. Experiments on the mice indicate that these IBs are not developed in absence of the TLR3 receptor. On the other hand, as it was mentioned, interferon $\beta$ affects HLA-G expression and inhibits its immune function $[16,17]$.
When some kinases (Tyrosine or Serine), including the p110 $\gamma$ subunit of class-I PI3-kinase (PIK3CG), IL2-inducible T-cell kinase (ITK), inhibitor of kappa light polypeptide gene enhancer in B-cells, kinase beta (IKBKB) and rapidly accelerated fibrosarcoma (RAF)-1 were under-expressed, observed a dampening in the inflammatory response. In addition, up-regulation of antiinflammatory genes such as cell proliferatory proteins and conserved helix-loop-helix ubiquitous kinase (CHUK), N-Ras and $\mathrm{K}$-Ras proto-oncogenes can influence the inflammatory response. Viral infected cells naturally upregulate some genes such as; IRF3, IRF7 and IFNB1, which could not stimulate an immune response with activation of the viral phosphoprotein. Furthermore, AKT activation (including IRF3 and IRF7) could change inflammatory macrophages to anti-inflammatory [18].

A low level of immune response is seen in victims of rabies. Rabies virus can escape immune responses using unique mechanisms and leads to death in non-vaccinated individual [2]. In general, it can be said that limitation of the inflammation by rabies virus infection may occur as follows: reduction of leukocyte, monocyte and macrophage insertion into the nerve 
tissue; maintaining the inability to pass the blood-brain barrier (BBB) to penetrate the CNS; minimizing the release of neurotoxic molecules that can maintain the function of the nervous system and host survival $[19,20]$.

\section{Adaptive Immune Response}

Second line of defense, which acts after innate immunity, is adaptive immunity. Formation of an adaptive immune response against a microorganism is always created in the peripheral lymphoid tissue. Nerve tissue is free of lymphatic organs.

\section{Cell-Mediated Immune (CMI) Response}

Cellular immunity plays a key role to eliminate viral invasion and inhibited the access of RV to the peripheral nervous system (PNS) at the beginning of infection. However, blood brain barrier is a big challenge for CMI. In addition, expression of the human leukocyte antigens (HLA)-G molecules on neuronal cells by the production of interferon beta leads to converting naïve human $\mathrm{T}$ cells to regulatory $\mathrm{T}$ cells and production of inhibitory cytokines such as interleukin 10 leads to cellular immunosuppression [11,21].

\section{Neutralizing Antibodies}

Humoral immune system plays an important role against the rabies virus. When RV enters to body of immune individuals, neutralizing antibodies can neutralize viral infectivity and prevent adsorption of virus to neuronal cells. Studies indicated that non-immune individuals cannot produce appropriate level of neutralizing antibodies when affected by RV, thus the virus enters to nerve. Actually, neutralizing antibodies is produced in peoples who received the vaccine and in people who survived the disease (rarely) [11].

\section{Apoptosis}

Neurotropic viruses also cause cell death due to apoptosis or necrosis. Apoptosis is the main defense mechanism against the cells infected with the virus, and it prevents the virus spread in the body. Apoptosis of the virus-infected cells are directed by the cytotoxic T cell and cytokines such as TNF- $\alpha$, perforin, granzyme and Fas-FasL (Fas Ligand) reaction. Instead of being a host defense mechanism for the elimination of infected neurons, apoptosis could be specifically inhibited by RABV as an immune subversive strategy. As a strategy for neuro-invasiveness of the street RV strains and progression through the nervous system, the virus suppresses early apoptosis in neuronal cells via up-regulation of AKT2 and AKT3 by G viral proteins and in turn, provides preapoptosis conditions in the immune cells via high representation of FAS Ligand [18]. In fact, there is an inverse correlation between the potency of a virus strain to invade the brain and induction of apoptosis [18]. Neurons, microglia and astrocytes also contribute to the viral proliferation via changing neuronal function [22].

\section{HLA-G Expression}

HLA-G1 molecule is expressed on the neurons due to stimulation of rabies virus. This molecule prevents the inflammatory function of natural killer (NK) and $\mathrm{T}$ cells in infectious conditions. In fact, the HLA-G molecule is attached to the inhibitory receptors of the $\mathrm{NK}$ and $\mathrm{T}$ cells and leads to the destruction of these cells. The inhibitory receptor KIR2DL4, NK cell-activating receptor with inhibitory potential, located on the NK cell and immunoglobulin inhibitory receptor (immunoglobulin like transcript (ILT)), such as ILT2 and ILT4 expressed on NK, TCD8+ and TCD4+ cells, are attached to the HLA-G molecule. On the other hand, HLA-G is able to develop tolerance-mediating dendritic cells by destructing the major histocompatibility complex (MHC) class II presentation pathway. Cytokines such as interferon beta also lead to the expression of the HLA-G molecule. In fact, following infection of the neuron with RV due to presence of HLA-G molecule on the neuron surface, occurs an adjustment of the immune response [21].

\section{Discussion}

The pathogenic agents should be able to inhibit and escape from immune system to cause chronic or fatal diseases. The virus is a lethal virus that leads to inhibition of the immune system responses using some mechanisms thus causes disease and death of the patient $[3,22,23]$. Studies showed that the components of intrinsic immunity as the first defense barrier cannot be activated quickly and stimulate the adaptive immunity for eradicating the disease. In addition, in this disease, performance of the immune system is highly limited due to special sensitivity and unusual structure of the nervous system. Therefore, due to limited immune responses, the disease progresses and the virus can continuously replicate and ultimately, acute and progressive encephalitis leads to the death of the patient [22]. Rabies is still prevalent in developing countries, affecting populations with limited access to health care. Human exposure to potentially rabies-infected animals is a public health concern worldwide. Thus, according to the WHO recommendation, individuals at-risk of rabies virus including rabies researchers, veterinarians, wildlife workers, dog breeders, animal handlers and veterinary students should receive rabies pre-exposure prophylaxis (PrEP) [24].

\section{References}

1. Lim PL, Barkham TM (2010) Serologic response to rabies preexposure vaccination in persons with potential occupational exposure in Singapore. International Journal of Infectious Diseases. 14(6): e511-e513.

2. Johnson N, Cunningham AF, Fooks AR (2010) The immune response to rabies virus infection and vaccination. Vaccine 28(23): 3896-3901.

3. Sridhar N, Kiran BS, Rudrapal M (2014) Management, Post-exposure Prophylaxis and Treatment of Dog Bites and Rabies Cases in a Tertiary Care Hospital.

4. Wunner WH (2003) Rabies virus. Rabies: Elsevier p. 23-77.

5. Malerczyk C, Briggs DJ, Dreesen DW, Banzhoff A (2007) Duration of immunity: an anamnestic response 14 years after rabies vaccination with purified chick embryo cell rabies vaccine. Journal of travel medicine14(1): 63-64.

6. Chacko K, Parakadavathu RT, Al-Maslamani M, Nair AP, Chekura AP, et al. (2017) Diagnostic difficulties in human rabies: A case report and review of the literature. Qatar medical journal 2016(2): 15. 
7. Koyuncu 00, Hogue IB, Enquist LW (2013) Virus infections in the nervous system. Cell host \& microbe 13(4): 379-393.

8. Yao C, Kaplan DH (2018) Langerhans cells transfer targeted antigen to dermal DC and acquire MHC-II in vivo. The Journal of investigative dermatology. J Invest Dermatol 138(7): 1665-1668.

9. Dietzschold B, Schnell M, Koprowski H (2005) Pathogenesis of rabies. The World of Rhabdoviruses: Springer p. 45-56.

10. Liard C, Munier S, Joulin-Giet A, Bonduelle O, Hadam S, et al. (2012) Intradermal immunization triggers epidermal Langerhans cel mobilization required for CD8 T-cell immune responses. Journal of Investigative Dermatology 132(3): 615-625.

11. Jochmans D, Neyts J (2017) The path towards effective antivirals against rabies. Vaccine 17: 31812-31821.

12. Gadre G, Satishchandra P, Mahadevan A, Suja M, Madhusudana S, et al. (2010) Rabies viral encephalitis: clinical determinants in diagnosis with special reference to paralytic form. Journal of Neurology, Neurosurgery \& Psychiatry 81(7): 812-820.

13. Jackson AC (2003) Rabies virus infection: an update. Journal of neurovirology 9(2): 253-258.

14. Hemachudha T, Sunsaneewitayakul B, Mitrabhakdi E, Suankratay C, Laothamathas J, et al. (2003) Paralytic complications following intravenous rabies immune globulin treatment in a patient with furious rabies. International Journal of Infectious Diseases 7(1): 76-77.

15. Johnson N, Un H, Fooks A, Freuling C, Müller T, et al. (2010) Rabies epidemiology and control in Turkey: past and present. Epidemiology \& Infection 138(3): 305-312.

16. Préhaud C, Mégret F, Lafage M, Lafon M (2005) Virus infection switches TLR-3-positive human neurons to become strong producers of beta interferon. Journal of virology 79(20): 12893-12904.
17. Menager P, Roux P, Megret F, Bourgeois JP, Le Sourd AM, et al. (2009) Toll-like receptor 3 (TLR3) plays a major role in the formation of rabies virus Negri Bodies. PLoS pathogens 5(2): e1000315.

18. Azimzadeh Jamalkandi S, Mozhgani SH, Gholami Pourbadie H, Mirzaie M, Noorbakhsh F, et al. (2016) Systems biomedicine of rabies delineates the affected signaling pathways. Frontiers in microbiology 7: 1688

19. Reinke SN, Resch L, Maingat F, Branton W, Jackson AC, et al. (2012) Metagenomic and metabolomic characterization of rabies encephalitis: new insights into the treatment of an ancient disease. The Journal of infectious diseases 207(9): 1451-1456.

20. Johnson N, Cunningham AF (2015) Interplay between rabies virus and the mammalian immune system. World Journal of Clinical Infectious Diseases 5(4): 67-76.

21. Lafon M, Prehaud C, Megret F, Lafage M, Mouillot G, et al. (2005) Modulation of HLA-G expression in human neural cells after neurotropic viral infections. Journal of virology 79(24): 15226-15237.

22. Fernandes ER, de Andrade Jr HF, Lancellotti CLP, Quaresma JAS, Demachki S, et al. (2011) In situ apoptosis of adaptive immune cells and the cellular escape of rabies virus in CNS from patients with human rabies transmitted by Desmodus rotundus. Virus research. 156(1-2): 121-126.

23. Zhugunissov K, Bulatov Y, Taranov D, Yershebulov Z, Koshemetov Z, et al. (2017) Protective immune response of oral rabies vaccine in stray dogs, corsacs and steppe wolves after a single immunization. Archives of virology 162(11): 3363-3670.

24. Organization WHO (2013) WHO expert consultation on rabies: second report. 